\title{
Regional variations in contraceptive use in Kenya: comparison of Nyanza, Coast and Central Provinces ${ }^{1}$
}

\author{
Murungaru Kimani, PhD \\ Senior Lecturer, Population Studies and Research Institute (PSRI) \\ University of Nairobi, Nairobi, Kenya. E-mail: murungaruk@uonbi.ac.ke \\ Milton Njeru \\ JHPIEGO, Nyeri, Kenya. \\ Dr. Gathari Ndirangu \\ DRH/Capacity Kenya, Nairobi, Kenya.
}

\begin{abstract}
This paper analyses the regional variations in contraceptive use between Central, Nyanza and Coast Provinces in Kenya among currently married, fecund women drawn from the 2008-09 Kenya Demographic and Health Survey (KDHS) data. Specifically the study examined the role of socio-economic, cultural and demographic factors in explaining these variations using both bivariate and logistic regression. The analysis confirmed the higher use of contraception in Central compared to Nyanza and Coast. Current use of modern contraceptive methods in Central is 70 percent compared with 39 percent and 37 percent for Nyanza and Coast respectively. The higher contraceptive use in Central is attributed to the better socio-economic and cultural environment compared with the other two provinces. Central Province has very few cases of women with no education, a much lower percentage in the poorest wealth (9.6) category and the highest proportion in monogamous unions (97.I). The higher socio-economic status and better cultural environment has in turn created a favourable environment for the use of contraception through the intervening variables of knowledge on family planning and fertility preferences. The logistic regression results suggest that differences in contraceptive use between the three provinces could be narrowed by increasing the level of education in Coast and overcoming traditional practices such as polygyny in both Nyanza and Coast. Although mortality is still important, its effect has declined. However, the unexpected finding that contraceptive use is higher in rural areas of Central and Nyanza Provinces suggests further research to understand what could be responsible for the reversal.
\end{abstract}

Keywords: Modern, contraceptive use, regions, variations

\section{Introduction}

The use of contraception has the potential to reduce maternal mortality and infant deaths through increased birth spacing and avoidance of higher-risk pregnancies and hence contribute to the attainment of International Conference on Population and Development (ICPD) goals and MDGs. It also has the potential to slow the rate of population growth, thereby contributing to a reduction of poverty and hunger (WHO, 20I0). The Ministries of Health in Kenya aim to increase contraceptive prevalence from the current 46 percent to 56 percent by the year 2015 (Ministry of Medical Services and Ministry of Public Health and Sanitation).

Although contraceptive use in Kenya has been increasing over the years, substantial regional differences have persisted. The 2008-09 Kenya Health Demographic and Health Survey (KDHS) shows that, while the prevalence of modern contraceptive use in Central and Nairobi Provinces is comparable to the levels in developed countries, it is extremely low in North Eastern Province, at only 3 percent among married women. Three possible explanations for these differences have been suggested, namely socio-economic, cultural and the impact of family planning programs (Clements et al. 2004). Evidence from research conducted in Kenya and elsewhere has provided empirical evidence to support these suggestions (Kimani and K'Oyugi, 2004).

In this study we use data from the 2008-09 $\mathrm{KDHS}$ to investigate regional variations in modern contraceptive use among married women at risk of becoming pregnant, to demonstrate the influence of socio-economic, demographic and cultural factors. The study focuses on Coast, Nyanza and Central Provinces, where there are substantial differences in the use of modern contraceptive methods among

I. We are grateful to the National Council for Population and Development (NCPD) for allowing us to publish this paper, a version of which was published as a working paper by the same organisation; also to ICF Macro for funding the authors in the further analysis of KDHS workshop in Nairobi when the draft paper was prepared. 
married women. Central Province has the highest contraceptive prevalence, at 63 percent, while Nyanza and Coast Provinces are much lower, at 33 percent and 30 percent respectively (KNBS et al. 20I0). Specifically, the study analyses socio-economic, cultural and demographic factors that may have contributed to the observed differences in contraceptive use in the three regions. We test the hypothesis that differences in these factors within the three regions could explain the variation in levels of modern contraceptive use. Although previous studies in Kenya (Njogu, 199I; African Population Policy Research Center, 1998; Magadi and Curtis, 2003; Kimani, 2007) have analysed the factors associated with levels and trends in contraceptive use in Kenya, few studies have focused on the regional differentials except Kimani and K'Oyugi, 2004 and Clements et al. 2004 . Indeed, lack of such studies is not only limited in Kenya. Cammack and Heaton (200I) observe that although the individual factors associated with contraceptive use are well documented, those focusing on regional variations are few and hence our understanding of such factors is limited.

The choice of these three provinces is justified by a number of considerations. The three regions fall within the 20 percent high-potential areas of Kenya in which the Kenya's population is concentrated. Previous research has also demonstrated the potential use of the analysis in the formulation of regional strategies for the enhancement of contraceptive use in these regions. Kimani and K'Oyugi (2004), for example, showed that promotion of education in the Coast and reduction of infant and child mortality in
Nyanza would enhance the use of contraception in these regions. In addition, both Nyanza and Central Provinces are comparable in terms of infrastructure, population distribution, religion and availability of services. For example, most of the populations in the two provinces reside in the rural areas. Population density is also high in both provinces. Similarly, results from Kenya Services Provision Assessment (KSPA) 2010 showed that 89 percent of the sampled health facilities in Central offered some type of temporary contraceptive methods, which is comparable to the 93 percent in Nyanza (NCAPD et al. 20I I). In contrast, these variables are largely different in Coast Province, which has a substantial proportion of Muslims compared with Central and Nyanza provinces, with their much higher proportions of Christians.

Figure I shows the conceptual framework that guided the study. This framework can be perceived as a component within the Bongaarts (1978) proximate determinants framework for the analysis of fertility. However, since the focus of this study is limited to contraception, we did not consider the other proximate determinants of fertility identified by Bongaarts. Instead, the framework for this study has introduced intervening variables that are considered to have a direct influence on the use of contraception. The assumption is that the background socioeconomic, cultural and demographic variables create environments that, through the intervening variables, either favour or restrain the practice of contraception.

\section{Figure 1: Conceptual Framework}

Social, economic, cultural
and demographic factors
(Independent Variables)
-Education
-Religion
-Rural/Urban residence
-Type of marriage
-Wealth status

\section{Data and methods}

Data for the study were obtained from the 2008-09 $\mathrm{KDHS}$, a nationally representative sample consisting of 8,444 women aged 15-49. The design of the survey and the specific questions that were covered are detailed in the KDHS 2008-09 report (KNBS and ICF Macro, 20I0). The analysis was limited to 3,708

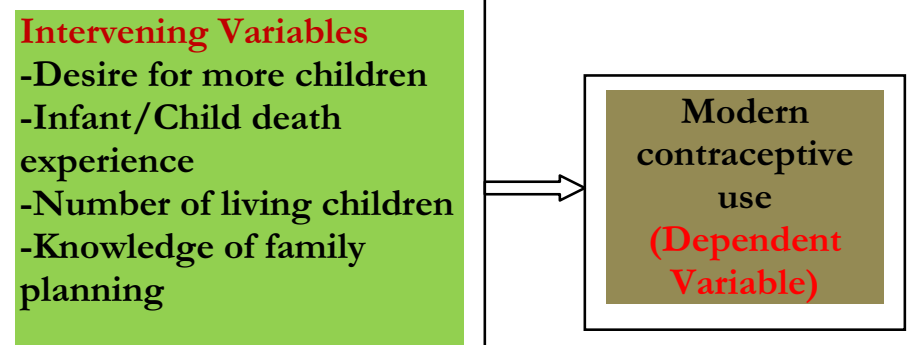

currently married women aged I5-49 at risk of becoming pregnant; that is, excluding women who were pregnant or infecund. The sample also excluded 65 cases, 64 because they did not have information on types of marriage and one other that lacked information on religion. All the analyses undertaken were based on the weighted sample.

The dependent variable for the study is current 
use of modern methods of contraception, including sterilisation. As observed by Dodoo (1995), modern methods of contraception, which are more effective than traditional methods, are the focus of most national family planning programmes, including in Kenya. Several socio-economic, cultural and intervening variables that have been found to be associated with contraceptive use and that are considered key explanatory variables for differences in contraceptive use were included, as indicated in the conceptual framework.

The key socio-economic variables include: residence, education and wealth index. Residence was categorised into rural and urban, while education was categorised into: none, primary and secondary and above. However, in Central and Nyanza two education categories, none and primary, were combined because of the limited number of cases of women with no education. Similarly, the wealth index was categorised into three categories: poorest, middle and wealthiest, rather than the usual wealth quintiles (poorest, poorer, middle, richer, richest) because of the few cases in Central among the poorest category.

Type of marriage (monogamous/polygynous) was used as a proxy for cultural differences among the three regions. To capture the influence of religion on contraceptive use, religious affiliation was categorised into: Christians, Muslims and Others/None.

Intervening variables include fertility preference, death of infant and/or child, number of living children and knowledge of modern family planning methods. Women were categorised by whether they desired more children or not. The former category included women who desired a child within two years or after two years, and also women who were undecided. Studies in Kenya, as elsewhere, have shown that women who desire no more children are more likely to use contraception (Kimani, 2007). The infant mortality variable categorised women into those who had lost at least one infant or child in the five years prior to the survey and those who had not. Women in the former category may be less likely to use contraception compared with those in the latter category because of replacement and insurance effects, as suggested by studies in Kenya and elsewhere (Kimani, 200I). Women who knew of fewer than seven modern contraceptive methods were categorised as having a low level of family planning knowledge, and women who knew of at least seven methods as having a high knowledge.

Both bivariate and multivariate analyses were performed using SPSS. Bivariate analysis was performed through cross tabulation to provide comparative associations between modern contraceptive use and the socio-economic, cultural and demo- graphic variables and intervening variables for the three regions and at the national level. Multivariate analysis was performed using logistic regression. Four logistic regression models, one for each of the regions and at the national level, were fitted and the effects of the various variables compared. However, both religion and family planning knowledge variables were excluded from the regressions. In Central and Nyanza, which are predominantly Christian, the samples included few Muslims. Furthermore, estimates based on models that excluded this variable at the national and Coast Province levels were similar. The knowledge variable was excluded because the results would have been difficult to interpret, as the effects could be in either direction. On the one hand, women could be expected to use contraception because of their knowledge of family planning methods, but on the other hand, women with family planning knowledge could have obtained this knowledge when they went to seek family planning services.

\section{Results}

Table I summarises the percent distributions among married, fecund, non-pregnant women for the three regions, Central, Coast and Nyanza, as well as at the national level, according to the study conceptual framework. Use of modern contraceptives is substantially higher in Central (70 percent) compared with Coast (37 percent) and Nyanza (39 percent), and the national level is 46 percent. The table also shows substantial regional differences in the distributions of the socio-economic, cultural, demographic and intervening variables.

The level of education among women is high in both Central and Nyanza Provinces and is comparable to the national level, but much lower in the Coast. In Central and Nyanza, very few women have no education, compared with 25 percent in the Coast and slightly over 9 percent at the national level. The level of household wealth is higher in Central compared with the other two regions. In Central, less than 10 percent of women are in the poorest wealth category compared with nearly 43 percent in Nyanza and 33 percent in Coast. The proportion of women residing in urban areas is much higher in Coast compared with the other two regions and the national level.

Cultural conditions in the three regions also differ. Polygynous marriages are more common in Nyanza, at 19 percent, compared with 3 percent in Central Province, which is also substantially lower than the national percentage, at 12 percent. In Central and Nyanza provinces, Christians predominate, at over 98 percent, while Muslims constitute 34 per- 
cent of the population in Coast.

Table I Percent distribution of currently married, fecund, non-pregnant women I5-49 in Central, Nyanza and Coast Provinces, and for Kenya, KDHS 2008-09 by background characteristics

\begin{tabular}{lllll}
\cline { 4 - 5 } Current use of modern contraception & Central & Nyanza & Coast & Kenya \\
\cline { 2 - 3 } & Percent & &
\end{tabular}

\begin{tabular}{lllll}
\hline Not using & 29.6 & 61.3 & 63.5 & 53.6 \\
\hline Currently using & 70.4 & 38.7 & 36.5 & 46.4 \\
\hline Type of place of residence & & & & \\
\hline Urban & 15.8 & 11.3 & 54.8 & 24.0 \\
\hline Rural & 84.2 & 88.7 & 45.2 & 76.0
\end{tabular}

\section{Education level}

None

Primary $^{\mathrm{a}}$

$62.9 \quad 69.8$

Sec +

37.1

30.2

25.0

9.2

$47.6 \quad 58.6$

\section{Wealth Terciles}

Poorest
Middle
Wealthiest
Type of marriage

\begin{tabular}{llll}
9.6 & 42.7 & 32.5 & 34.2 \\
\hline 52.2 & 36.8 & 16.1 & 32.9 \\
38.2 & 20.5 & 51.4 & 32.9
\end{tabular}

\begin{tabular}{|c|c|c|c|c|}
\hline Monogamous & 97.1 & 81.3 & 88.0 & 88.3 \\
\hline Polygynous & 2.9 & 18.7 & 12.0 & 11.7 \\
\hline \multicolumn{5}{|l|}{ Religion } \\
\hline Christians & 98.7 & 98.3 & 56.7 & 90.3 \\
\hline Muslims & & & 34.1 & 7.0 \\
\hline None/Other ${ }^{b}$ & 1.3 & 1.7 & 9.2 & 2.7 \\
\hline \multicolumn{5}{|l|}{ Age } \\
\hline $15-24$ & 17.5 & 31.9 & 32.1 & 23.7 \\
\hline $25-34$ & 41.4 & 43.1 & 44.3 & 44.8 \\
\hline $35+$ & $4 \mathrm{4I.I}$ & 25.0 & 23.7 & 31.5 \\
\hline \multicolumn{5}{|c|}{ Desire for children } \\
\hline Wants more & 36.5 & 51.6 & 61.5 & 46.3 \\
\hline Does not & 63.5 & 48.4 & 38.5 & 53.7 \\
\hline \multicolumn{5}{|c|}{ Whether any child died in last 5 years } \\
\hline No death & 94.9 & 85.7 & 93.0 & 92.6 \\
\hline Had a death & 5.1 & 14.3 & 7.0 & 7.4 \\
\hline \multicolumn{5}{|c|}{ Living children } \\
\hline $0-1$ & 19.5 & 19.8 & 26.2 & 18.3 \\
\hline $2-3$ & 45.1 & 40.6 & 38.7 & 40.5 \\
\hline $4+$ & 35.4 & 39.6 & 35.2 & 41.2 \\
\hline \multicolumn{5}{|l|}{ FP knowledge } \\
\hline Low & 8.6 & 32.0 & 38.2 & 33.1 \\
\hline High & 91.4 & 68.0 & 61.8 & 66.9 \\
\hline Total \% & 100 & 100 & 100 & 100 \\
\hline Total N & 416 & 625 & 307 & 3708 \\
\hline
\end{tabular}

\footnotetext{
a Includes no education in Central and Nyanza

b Includes Muslims in Central and Nyanza
} 


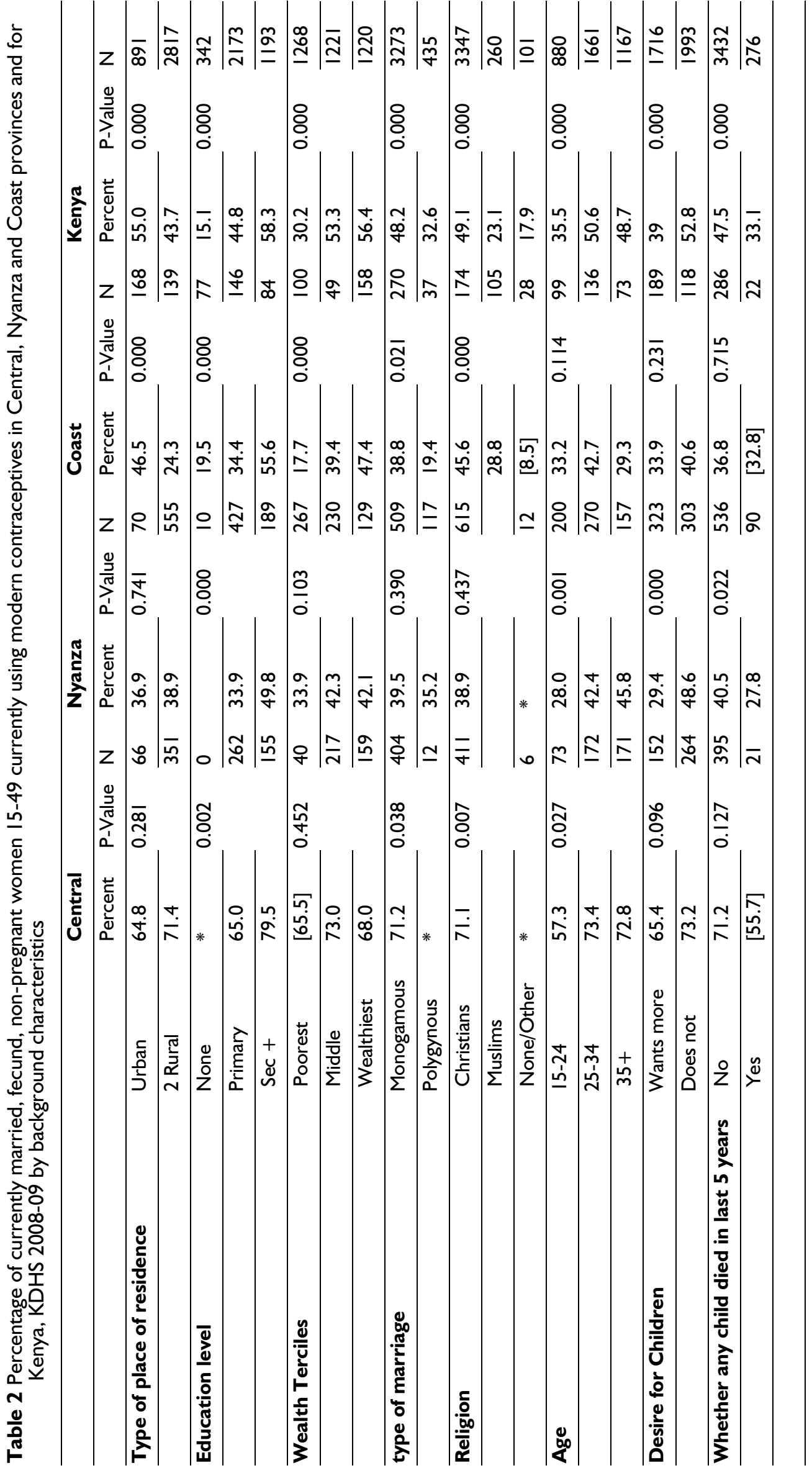




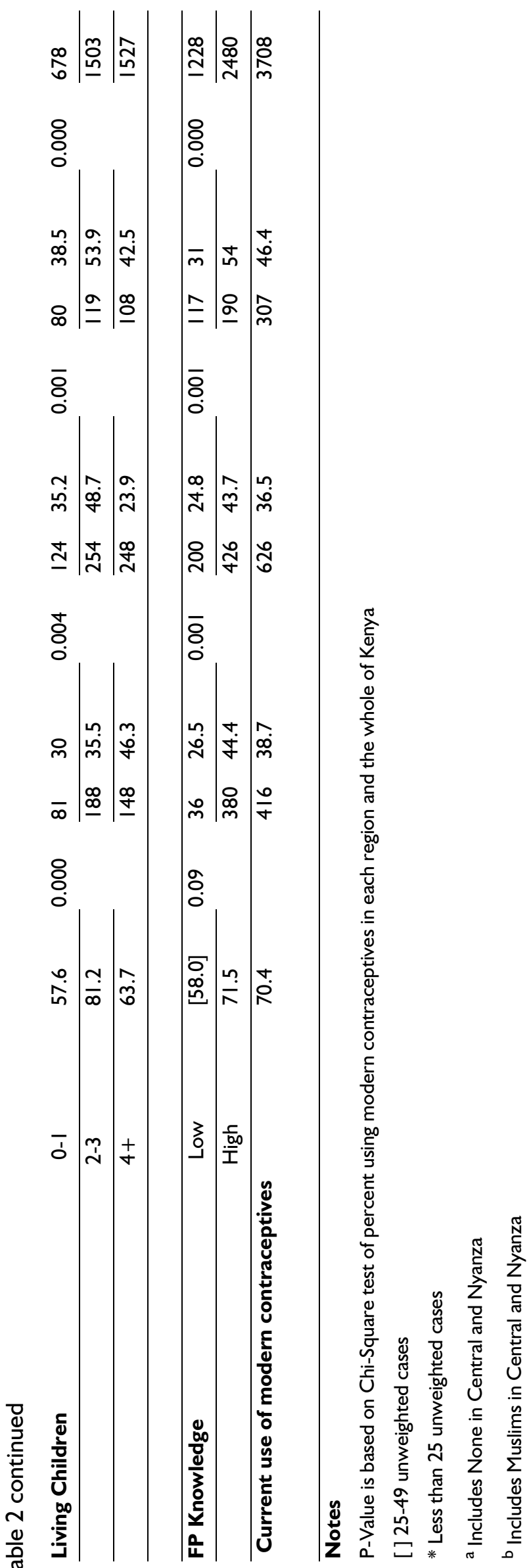

Compared with Coast and Nyanza Provinces, survey respondents in Central Province are older, with 4I percent of women aged 35 or older compared with 25 percent in Nyanza and 24 percent in Coast. In all three regions, the majority of women have 2-3 living children, while at the national level the proportion with 4 or more living children is marginally higher.

Finally, Table I shows differences in the regional distributions in the intervening variables. In both Nyanza and Coast Provinces, more than half of women desire to have an additional birth, at 52 percent in Nyanza and 62 percent in Coast compared with 37 percent in Central. The proportion of women who have experienced the death of at least one child in the five years preceding the survey is more than double in Nyanza compared with the other two regions and with the national level. Coast Province has the highest proportion of women who desire to have additional children, while Central had the lowest proportion. Knowledge of contraception is highest among women in Central Province, at 91 percent, while in the other two provinces the levels are below 70 percent, similar to the national average.

Table 2 summarises the association between contraceptive use and socio-economic, cultural and demographic and intervening variables in each province, as well as the national level. Contraceptive use increases with women's education and wealth, in the three regions and at the national level. At the national level, for example, contraceptive use among women with a secondary and higher level of education is 58 percent compared with 15 percent among women with no education. Similarly, contraceptive use is 56 percent among women in the wealthiest category compared with 30 percent in the poorest. These patterns are similar among the three regions. Although contraceptive use is higher in the urban areas of Coast Province and at the national level, the reverse is the case in Central and Nyanza, although these associations are not statistically significant. In Central, contraceptive use is 71 percent in rural areas compared with 65 percent in urban areas. By type of marriage, in the three regions and at the national level, use of modern contraception is significantly higher among women in monogamous unions, except in Nyanza, where the association is not statistically significant. Also, contraceptive use is higher among Christians than among Muslims.

Concerning the association between contraceptive use and the intervening variables, in the three regions and nationally contraceptive use is higher among women who do not desire more children. However, this relationship is not significant in Coast Province. Similarly, among women who experienced a child death in the five years preceding the survey, a 
lower proportion used contraception compared with women who did not experience a recent child death, although this relationship is only significant at the national level and in Nyanza. In all three regions and nationally, contraceptive prevalence is higher among women who know at least seven modern methods of contraception. Generally, contraceptive use is highest among women age 25-34 and among women with 2-3 living children, except in Nyanza, where contraceptive use increases with women's age and number of living children.

Table 3 summarises the logistic regression results. After controlling for the other factors in the model, socio-economic and cultural factors are significantly associated with contraceptive use at the national level. Use of contraception increases with levels of women's education, and the same pattern is observed with respect to the wealth index. Contraceptive use is also higher among women in monogamous marital unions. Also, after controlling for the effects of the other factors in the model, the intervening variables are significantly associated with contraceptive use at the national level. Women who do not desire another birth are more likely to use contraception. Contraceptive use is higher among women who did not experience a child death in the five years before the survey. Use of contraception is highest among women aged 25-34 and those with 23 children. Living in urban areas of Kenya, however, is not associated with higher use of contraception.

Similar to the bivariate analysis, in the multivariate analysis the factors associated with contraceptive use in the three regions are not as strong as at the national level. Indeed, in Central region, where the level of contraceptive use is comparable to developed countries, contraceptive use is only associated with women's education, type of marriage and age. Contraceptive use is higher among women with secondary and above education compared with those with none/primary, higher among women in monogamous unions compared with women in monogamous unions and higher among women age 35 or older compared with the reference group (women age 15-24). In Nyanza Province contraceptive use is only associated with women's education and fertility preferences. In Coast Province contraceptive use is only significantly associated with number of living children, with use being highest among women with 2-3 children.

\section{Discussion}

This study confirms the existence of substantial regional variations in contraceptive use in Kenya. Consistent with previous DHS results, it shows a higher level of modern contraceptive use in Central Province compared with both Coast and Nyanza Provinces, and also above the national average. Overall, the results reveal that women in Central Province are of higher socio-economic status compared with women in the other two regions. Central Province has very few cases of women with no education and also has a much lower percentage of women in the poorest wealth category compared with the other two provinces studied. Similarly, Central seems to be less culturally conservative, as reflected in the higher proportion of married women in monogamous unions compared with the other two regions.

The better socio-economic and cultural environment in Central Province seems in turn to have a positive impact on the intervening variables. Central Province has the most favourable environment for the use of contraception, as suggested by the higher proportion of women who do not desire to have another birth and the higher level of knowledge about family planning compared with the other two provinces. Other studies of Kenya have reported similar findings (Kimani and K'Oyugi, 2004).

The analysis shows that contraceptive use generally increases with higher socio-economic status, less cultural conservatism and a favourable environment for the use of contraception, as reflected by the intervening variables. Women in monogamous unions who can be considered less conservative are associated with higher levels of contraceptive use, except in Nyanza. This is consistent with the observed regional differences in contraceptive use, particularly between Central and Nyanza, and especially in view of the larger proportion of monogamous marriages in Central. Women who do not desire another birth are also more likely to use contraception, except in the Coast. This association is consistent with the observed regional differences, particularly between Central and Coast. Finally, the greater use of contraception among women with more knowledge of contraceptives in Central compared with the other two regions is also consistent with the observed regional differences.

The logistic regression results show that socioeconomic development and cultural conditions are important factors in the use of contraception in Kenya. This finding is consistent with demographic transition theory, which suggests that socio-economic development is the driving force for fertility control. Socio-economic development in turn seems to have shaped attitudes towards family planning and fertility preferences in Kenya. 


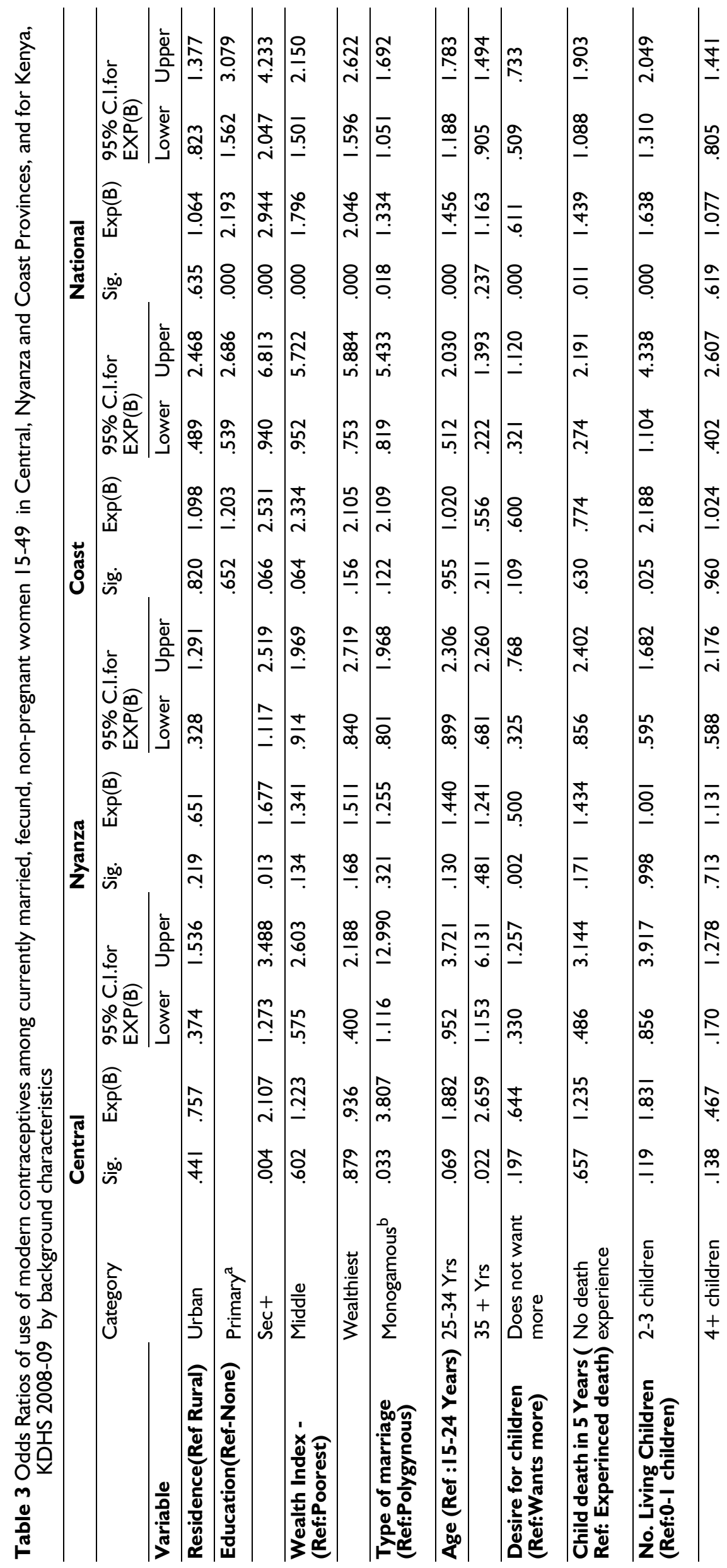




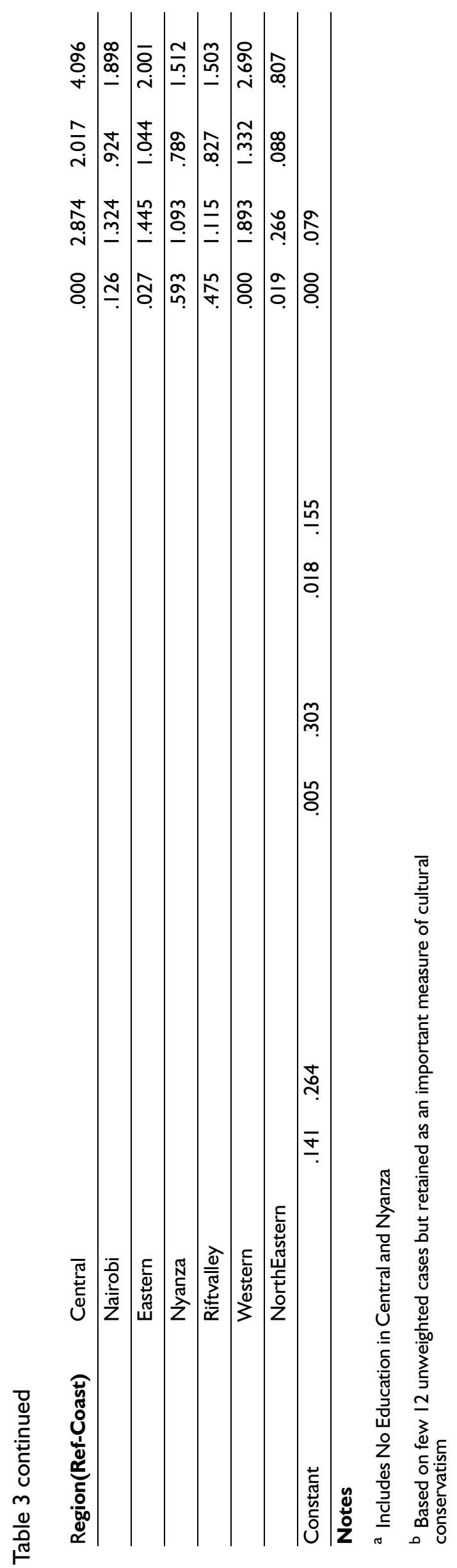

As expected, women who do not desire to have another birth are more likely to use contraception than other women. The observation that use of contraception is highest among women with 2-3 children suggests that Kenyan women are not only desiring smaller families but also taking deliberate action to realise their fertility preferences by using modern contraceptive methods.

An unexpected result is that urban residence is not associated with higher contraceptive prevalence, net of other factors. Although contraceptive use is higher in urban areas than rural areas of Coast Province and at the national level, this is not the case in Central and Nyanza, although the associations are not statistically significant. This finding is inconsistent with previous analyses that have suggested the presence of lower fertility and higher contraceptive use in urban areas. Our preliminary analysis at the regional level seems to imply that urban areas do not necessarily provide a better environment for the use of contraception. Related to this finding, the 2008-09 KDHS showed that infant mortality was higher in urban areas compared with rural areas at the national level (KNBS et al. 20I0).

The persistence of regional differences in contraceptive use in the presence of statistical controls seems to imply the omission of some key variables. For example, previous studies (Population Council, 1998; Kimani, 2007) have shown that both respondents' approval of family planning and discussions with the spouse about family are important, although these variables are not available in the 2008-09 KDHS.

The logistic regression results for the three regions show that the effects of the various factors on contraceptive use differ among these regions. Education is an important predictor of contraceptive use both in Central and Nyanza. This seems to suggest that increases in the levels of education in the Coast would result in higher levels of contraceptive use. The persistence of cultural practices such as polygyynous marriages could also be contributing to regional differences, as this practice is still prevalent in Nyanza and the Coast. The effect of the type of marriage still persists (results not shown) even when religion is controlled for, hence confirming its independent effect. However, the importance of desired family size on contraceptive use in the Coast could also be contributing to the differences. The proportion of women who desire to have another birth is substantial in this region in particular.

The lower use of contraception at the national level observed among women who experienced a child death in the five years before the survey confirms that child mortality is still an important factor associated with the demographic transition in Kenya. 
However, its importance seems to have been declining, particularly at the regional level. In our study, in the three regions the experience of a child death is not significantly associated with contraceptive use, after controlling for the other factors.

\section{Conclusion}

The better socio-economic and cultural environment in Central Province could be responsible for the higher use of contraception observed in this province in comparison with Nyanza and Coast, and hence with the observed regional differences in the use of modern methods of contraception. The higher use of contraception in Central arises from the favourable environment for the use of family planning, as reflected in the intervening variables. As the analysis reveals, the motivation to have additional births and the experience of child deaths are lower in Central than in the other two provinces, while women in the Central Province also have the highest levels of knowledge on family planning methods. Thus continued progress in socio-economic development, abandonment of traditional practices and reduction of infant and child mortality would promote the level of contraceptive use at the national level, and also reduce regional differences in contraceptive use.

The other important conclusion is that contraceptive use has spread even among women with small families, which suggests increasing acceptance of the small family norm in Kenya. Our final observation relates to the finding that urban areas in Kenya may no longer provide a better environment for contraceptive use compared with rural areas. The fact that in some regions use of contraception is higher in rural areas compared with urban areas suggests the need for further research to understand what could be responsible for this apparent reversal.

\section{References}

Bongaarts, J. 1978. "A Framework for Analyzing the Proximate Determinants of Fertility."Population and Development Review. Vol. 4, I: I05-I 32.

Cammack M. and Tim B. Heaton. 200I. Regional Variation in Acceptance of Indonesia's Family Planning Program. Population Research and Policy
Review, Vol. 20, No. 6: 565-585.

Clements, S., Baschieri, A., Hennink, M., Madise, N. and Stephenson R . 2004. Explaining Areal Variations in Modern Contraceptive Use in East Africa [Online]. Available from: $h t t p: / /$ paa2004.princeton.edu/ download.asp?submissionld $=40164$.

Dodoo, F. N. A. 1995. "Explaining Contraceptive Use Differences: Do Men Play a Role?" African Population Studies. Vol I0, I : 54-93.

Kenya National Bureau of Statistics (KNBS) and ICF Macro. 2010. Kenya Demographic and Health Survey 2008-09. Calverton, Maryland: KNBS and ICF Macro.

Kimani , M. 2007. Trends in Contraceptive Use in Kenya, 1989-1998: "The Role of SocioEconomic, Cultural and Family Planning Factors."African Population Studies. Vol. 21,2: 321.

Kimani , M. and B.O. K' Oyugi, B.O. 2004. "Regional Variations in the Use of Contraception in Kenya." Genus. Vol. LX,2: 33-53.

Kimani, M. 200I. "Behavioural Effects of Infant and Child Mortality in Kenya." African Journal of Reproductive Health. 5,3-?)

Magadi, M.A. and S.L. Curtis. 2003. Trends and Determinants of Contraceptive Method Choice in Kenya. 2003. Studies in FP 34(3): I49-159.

National Bureau of Statistics (KNBS) and ICF Macro. 20I I. Kenya Service Provision Assessment Survey 20 10. http://www.measuredhs.com.

National Coordinating Agency for Population and Development (NCAPD), Ministry of Medical Services (MOMS), Ministry of Public Health and Sanitation (MOPHS) Kenya

Njogu W. I99I. Trends and Determinants of Contraceptive Use in Kenya. Demography, Vol. 28, No. I: 83-99.

The African Population Policy Research Center. 1998. Fertility Decline in Kenya: Levels, Trends and Differentials. Population Council.

World Health Organization. 2010. Sexual and Reproductive Health Package of Interventions for Family Planning, Safe Abortion Care, Newborn and Child Health. [Online]. Available from: http:// whqlibdoc.who.int/hq/2010/ WHO_FCH_10.06_eng.pdf. 